\title{
Modes de caractérisation de fibres optiques unimodales
}

\author{
J. Auge, J. P. Pocholle et J. Raffy \\ Thomson-CSF, Laboratoire Central de Recherches, Domaine de Corbeville, B.P. 10, 91401 Orsay, France
}

(Reçu le 3 juin 1983, accepté le 8 septembre 1983)

\begin{abstract}
Résumé. - On décrit les différents modes de caractérisation permettant d'évaluer les propriétés de transmission de fibres optiques unimodales. Les méthodes présentées déterminent l'atténuation spectrale, le profil d'indice sur préforme ou sur fibre, la longueur d'onde de coupure, la dimension du mode et la dispersion chromatique.
\end{abstract}

\begin{abstract}
This paper deals with the measurement techniques developed for evaluating transmission characteristics of single mode fibres. The measurements include the determination of loss, preform and fibre refractive index profiles, cut off wavelength, spot size, chromatic dispersion.
\end{abstract}

\section{Introduction.}

Les fibres optiques unimodales représentent un des éléments clés des lignes de transmission constituant les futures grandes artères de communication [1].

L'aspect pour le moins paradoxal est que ce support de transmission destiné à véhiculer des informations sans altération de celles-ci peut également être inséré dans des montages optiques le transformant en élément capteur [2-4], sensible plus ou moins sélectivement à certains paramètres physiques d'environnement (température, pression acoustique, champ magnétique, ...) ou au mouvement (gyromètre, accéléromètre, ...).

Les caractéristiques de telles fibres peuvent également contribuer au développement de nouvelles architectures de bus optiques à distribution en anneaux ou hybrides, autorisant un nouveau champ d'application aux éléments d'optique dite intégrée $[5,6]$.

De telles fibres constituent donc un futur support adapté au développement des communications en général et de la télématique interactive en particulier avec l'avènement de la numérisation des signaux vidéo.

Certains paramètres physiques caractérisant de tels guides présentent une importance particulière pour la conception, la réalisation ou l'insertion de ce type de fibre dans les systèmes de communication : haut débit - grand pas de régénération. Dans cette perspective, nous décrivons les différents modes de caractérisation en les reliant aux deux principaux bilans pouvant affecter la transmission de signaux, à savoir :

- Le bilan énergétique lié à l'atténuation de la ligne, à la puissance optique couplée (avec une borne supérieure associée à l'apparition d'effets non linéaires), à la sensibilité et aux caractéristiques de bruit du récepteur.

- Le bilan fréquentiel dépendant des caractéristiques de dispersion de la ligne, des propriétés spectrales de la source.

A cette fin, nous analysons l'importance des paramètres devant être mesurés selon le niveau où se situe la caractérisation dans la chaîne de réalisation du composant monomode.

Dans cette chaîne, nous considérons les phases liées : à l'étude au niveau du laboratoire, au développement à caractère industriel, aux étapes associées à la mise au point d'une structure de câble et à l'emploi de l'élément monomode au niveau du système.

\section{Paramètres optogéométriques.}

Une fibre optique à échelon d'indice est dite monomode si la condition :

$$
V=\frac{2 \pi a}{\lambda} \sqrt{2 n_{1} \Delta n} \leqslant 2,405
$$

est réalisée, avec $a$ : rayon de cour, $\lambda:$ longueur d'onde de source, $n_{1}$ : indice optique du matériau constituant le coeur de la fibre, $\Delta n$ : différence d'indice entre les matériaux composant le coeur et la gaine optique.

Sur la figure 1, nous présentons la variation du couple $a, \Delta n$ satisfaisant la relation 1 aux longueurs d'onde de coupure (i.e. $\left.V_{\mathrm{c}}=2,405\right) \lambda_{\mathrm{c}}=1$ et $1,2 \mu \mathrm{m}$. 


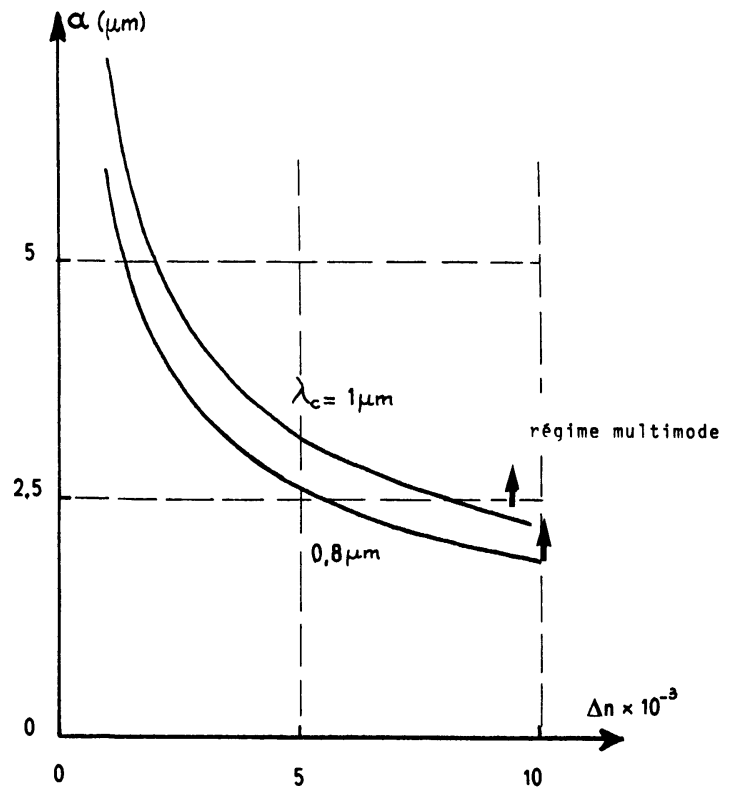

Fig. 1. - Conditions sur les paramètres $a$ (rayon de cour), $\Delta n$ (différence d'indice) déterminant le régime monomode en fonction de $\lambda$.

[Design diagram determining the monomode conditions at 1 and $1.2 \mu \mathrm{m}$.]

En fait, selon les procédés de fabrication mis en œuvre, des défauts de profil d'indice peuvent modifier cette condition déterminant le domaine de validité de la propagation monomode. Il est toutefois possible de définir une fibre à échelon d'indice équivalente. A titre d'exemple, nous avons porté sur la figure 2 un

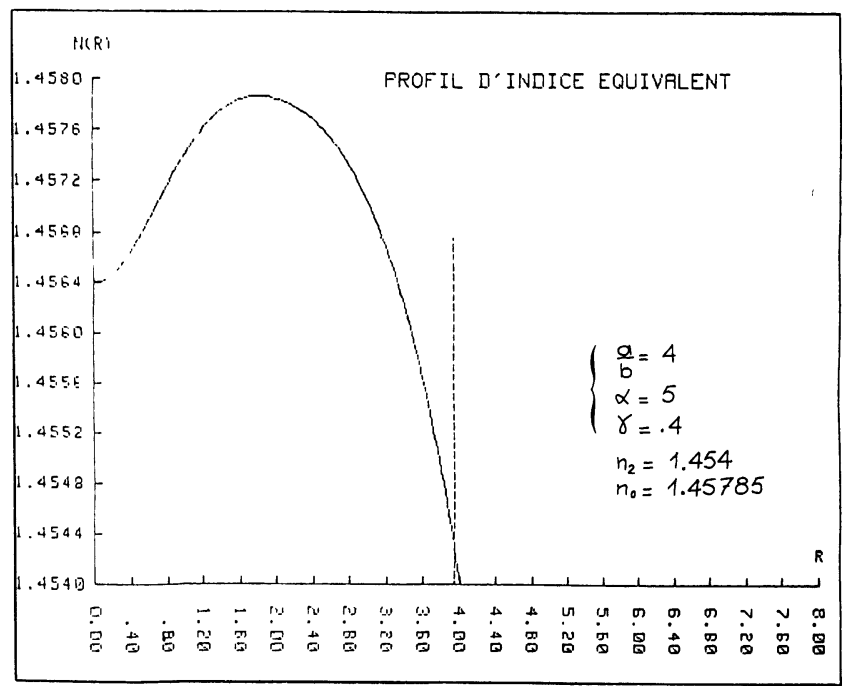

Fig. 2. - Comparaison entre le profil d'indice perturbé $n^{2}(R)=n_{0}^{2}\left[1-2 \Delta R^{\alpha}-2 \gamma \exp \left(-R^{2} \frac{a^{2}}{b^{2}}\right)\right] R=r / a$, $a$ : rayon de cour, $\Delta \simeq \Delta n / n_{1}$ et définition de la fibre à échelon équivalente.

[Determination of the equivalent step index parameters from the perturbed refractive index profile.] profil d'indice analytique correspondant aux deux principales perturbations pouvant être observées sur les fibres optiques (présence d'un gradient et d'un trou d'indice au centre). Par l'emploi d'une méthode de perturbation, il est possible de définir la fibre à échelon d'indice équivalente. La présence de tels défauts, si leur reproductibilité n'est pas assurée, va donc se traduire par une variation des paramètres optogéométriques équivalents, donc par une modification de la longueur d'onde de coupure et de l'extension radiale du mode.

Afin de contrôler ou de minimiser l'influence de tels défauts sur la propagation, il est souhaitable de disposer d'un montage de mesure du profil d'indice sur fibre ou sur préforme et qui soit non destructif dans son principe.

Différentes techniques de mesures ont été développées permettant d'accéder au profil d'indice sur fibres $[7,8]$. Ces mesures sont cependant limitées à un relevé du profil d'indice par prélèvement d'échantillons aux extrémités de la fibre réalisée. D'autre part, les dimensions de cœur des fibres optiques unimodales sont typiquement de quelques microns (Fig. 1) et les techniques de mesures fondées sur l'analyse du champ proche réfracté (7) ne peuvent résoudre avec fidélité les défauts du profil, puisque ce mode de caractérisation voit sa résolution limitée par le diamètre du faisceau d'analyse (compris entre 0,5 et $1 \mu \mathrm{m}$ ) de la face d'entrée de la fibre et par l'ouverture de l'optique collectant la lumière réfractée.

On peut accroître la résolution et affiner les mesures du profil d'indice en opérant sur la préforme. Dans ce cas, l'objet à analyser est de plus grande dimension et des méthodes de mesures non destructives peuvent être développées [7,9].

Parmi ces méthodes, mentionnons celles exploitant la déviation des rayons. Elles sont fondées sur les lois de l'optique géométrique paraxiale. La préforme est immergée dans une cuve remplie d'un liquide dont la valeur de l'indice $n_{2}$ est proche de celui associé au matériau constituant la gaine optique.

$\mathrm{Si}$ on suppose que la préforme présente une symétrie cylindrique, on peut l'identifier à une lentille cylindrique dont l'indice varie radialement. Ainsi, tout faisceau lumineux éclairant transversalement la préforme va subir une déviation. Si nous appelons $\Phi(t)$ l'angle de déviation en fonction de la position du faisceau incident par rapport à l'axe de la préforme (Fig. 3), nous pouvons remonter au profil d'indice radial en effectuant l'opération,

$$
n(r)-n_{2}=-\frac{n_{2}}{\pi} \int_{\mathrm{r}}^{\infty} \frac{\Phi(t)}{\sqrt{t^{2}-r^{2}}} \mathrm{~d} t .
$$

Le contrôle de la position du point $t$ est obtenu à l'aide d'un miroir tournant et le faisceau défléchi par la préforme est collecté par la lentille $\mathrm{L}_{2}$ et analysé par un détecteur de position. La lentille $L_{1}$ et le système afocal permettent d'ajuster la résolution 


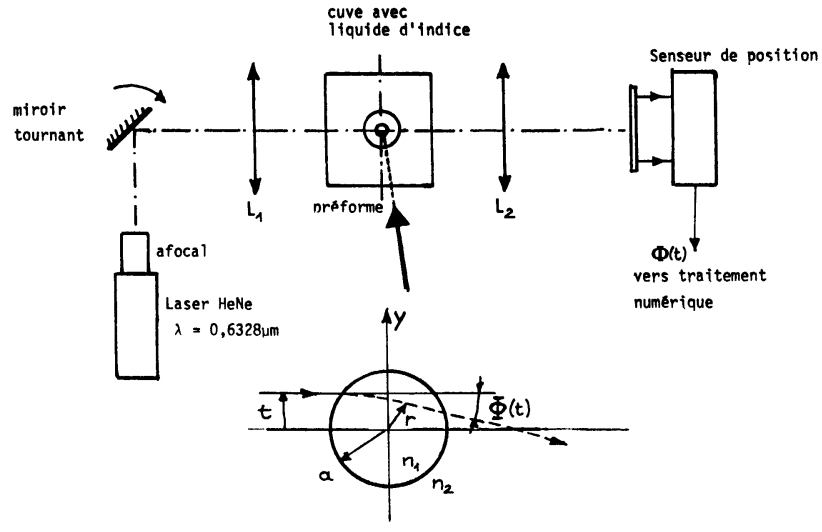

Fig. 3. - Mesure du profil d'indice sur préforme par la méthode de déviation du rayon.

[Index profile measurement of monomode preform using the beam deflection method.]

spatiale en jouant sur la dimension de la tache lumineuse au point de focalisation situé au niveau du cœur de la préforme.

La seconde méthode consiste à éclairer uniformément en lumière blanche à l'aide d'un faisceau collimaté la préforme immergée.

PROFIL D'INDICE SUP PREFORME

REFERENCE : CN62B
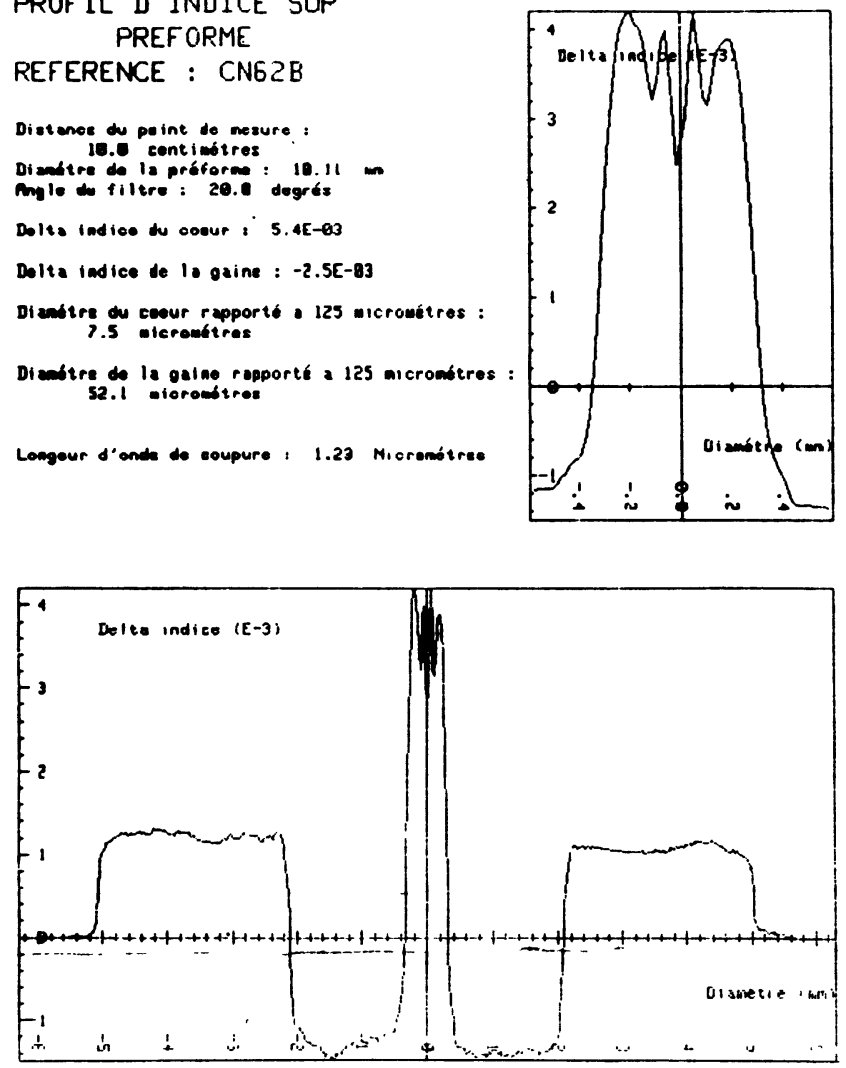

Fig. 4. - Résultat de mesure du profil d'indice sur préforme et projection des paramètres optogéométriques sur fibre.

[Typical index profile on preform and equivalent optogeometrical parameters on fibre.]
Une barrette de photodiodes enregistre la distribution d'intensité dans un plan situé dans la gaine optique de la préforme; un objectif établissant la conjugaison entre ce plan et la barrette de photodiodes.

L'enregistrement de la distribution de la densité du flux lumineux dans le plan d'analyse ainsi que l'application de la conservation du flux permettent de remonter à la fonction de déflexion $[7,10,11]$.

De telles méthodes de mesures ou leurs variantes déterminent les zones d'homogénéité des paramètres optogéométriques et définissent la partie de la préforme pouvant être fibrée tout en prévoyant les caractéristiques de transmission de la fibre (rayon de cour $a$, $\left.\Delta n, \lambda_{\mathrm{c}}\right)$. Un exemple de mesure sur préforme et la projection des paramètres sur fibre sont présentés sur la figure 4.

\section{Atténuation spectrale.}

L'atténuation spectrale est un paramètre important dans la caractérisation d'une fibre puisqu'il permet de contrôler et de qualifier un programme de dépôt ou une opération de fibrage. C'est également un facteur prépondérant pour projeter les portées d'une liaison monomode. La mesure consiste à effectuer l'enregistrement du flux lumineux $I_{1}$ en sortie d'une grande longueur de fibre $Z_{1}>2 \mathrm{~km}$ en fonction de la longueur d'onde $\lambda$.

Afin de s'affranchir de la fonction de transfert du couple source-détection, on réalise un second enregistrement $I_{0}$ sur une courte longueur de fibre $Z_{0}$ dénommée référence (Fig. 5).

Le coefficient d'atténuation linéique en $\mathrm{dB} / \mathrm{km}$ est alors obtenu en appliquant la relation :

$$
\begin{aligned}
\alpha_{\mathrm{dB} / \mathrm{km}}=\frac{10}{Z_{1}-Z_{0}} \log \left(\frac{I_{0}}{I_{1}}\right) & \simeq \\
& \simeq \frac{10}{Z_{1}(\mathrm{~km})} \log \left(\frac{I_{0}}{I_{1}}\right) .
\end{aligned}
$$

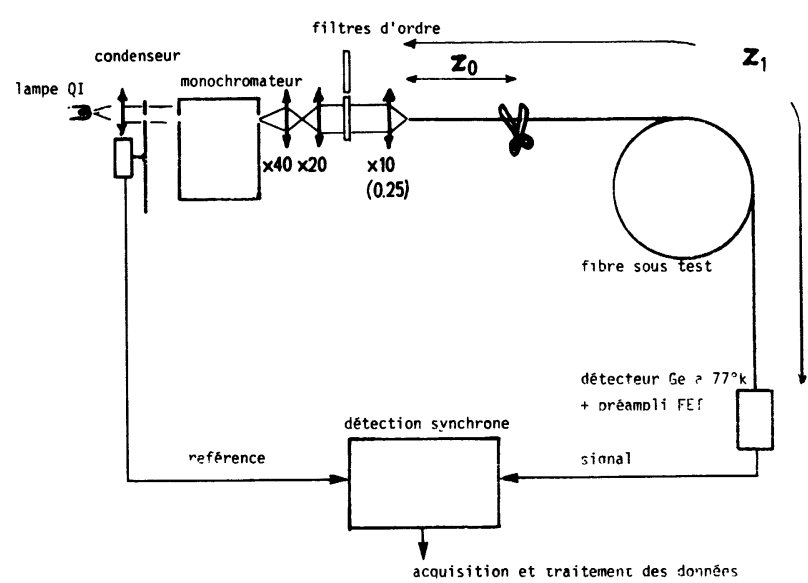

Fig. 5. - Schéma de montage de la mesure d'atténuation spectrale.

[Schematic representation of the spectral loss measurement.] 
Afin de minimiser les erreurs de mesures lorsque le coefficient d'atténuation est faible, on a intérêt à réaliser la mesure sur une grande longueur de fibre. (Rappelons que $0,2 \mathrm{~dB}$ correspond à un facteur de transmission de $95,5 \%$.)

Un résultat de mesure type est présenté sur la figure 6.

On peut sur cette courbe déterminer la longueur d'onde de coupure effective $\lambda_{\text {ceff }}$, ce pic d'atténuation étant le reflet d'un filtrage des modes ne satisfaisant pas la condition 1

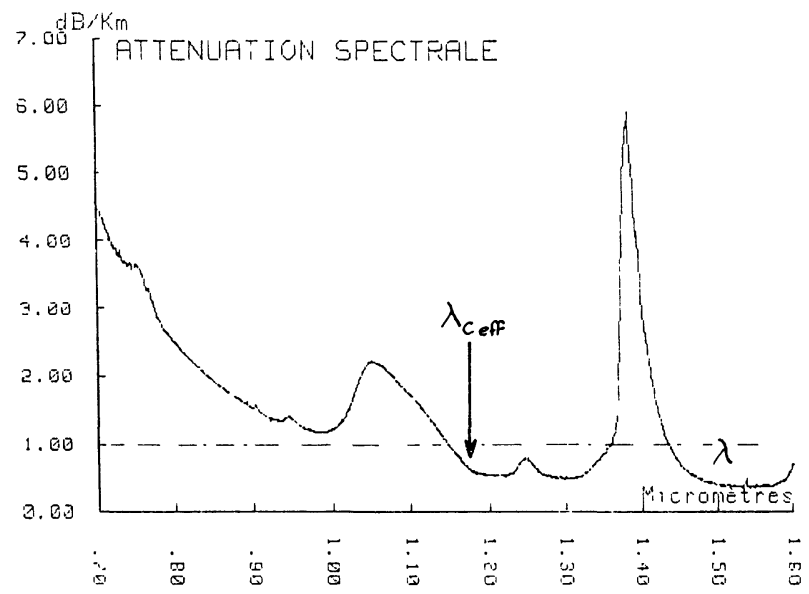

Fig. 6. - Courbe d'atténuation spectrale sur fibre optique unimodale.

[Spectral attenuation of monomode fibre.]

\section{Dimension du mode.}

Une fibre optique est principalement caractérisée par la dimension du mode. Ce paramètre permet d'appréhender les pertes en transmission associées aux opérations d'épissurage, de connexion, ou de déterminer la sensibilité de la fibre aux courbures et microcourbures.

A partir de la dimension du mode, il est également possible de concevoir la micro-optique permettant d'adapter les champs du mode de la source utilisée dans le système à celui de la fibre.

Différentes techniques de mesure ont été récemment proposées [12-14]. Dans l'approximation gaussienne du champ associé au mode fondamental, on peut relier celui-ci aux paramètres optogéométriques de la fibre équivalente par la relation [15-16]

$$
\begin{aligned}
\omega_{0} & =a_{\mathrm{e}}\left[0,65+0,434\left(\frac{\lambda}{\lambda_{\mathrm{c}}}\right)^{3 / 2}+0,0149\left(\frac{\lambda}{\lambda_{\mathrm{c}}}\right)^{6}\right] \\
V_{\mathrm{e}} & =2,405 \frac{\lambda_{\mathrm{c}}}{\lambda} . \\
\Delta n_{\mathrm{e}} & =5,05 \times 10^{-2}\left(\frac{\lambda_{\mathrm{c}}}{a_{\mathrm{e}}}\right)^{2} .
\end{aligned}
$$

Dans ces conditions, une mesure du paramètre $\omega_{0}=f(\lambda)$ permet de définir la fibre équivalente à échelon d'indice $\left(a_{\mathrm{e}}\right.$ : rayon de cour équivalent, $\Delta n_{\mathrm{e}}$ : différence d'indice équivalente) quel que soit le profil d'indice réel.

Dans cette perspective, nous avons développé une méthode de mesure fondée sur l'analyse du champ lointain par l'intermédiaire de masques. Le flux recueilli en fonction du déplacement de ceux-ci permet de remonter à la dimension du mode dans l'approximation gaussienne du champ.

Deux configurations de masques peuvent être employées. La première est constituée de barres alternativement transparentes et opaques (Fig. 7a) qui se déplacent dans le champ lointain, la seconde est composée d'une lame de rasoir que l'on déplace par rapport à la fibre (Fig. $7 b$ ). Une optique collecte le flux, issu du masque explorant le champ lointain. Cette intensité est ensuite focalisée sur un photodétecteur. A partir des mesures donnant les variations d'intensité en fonction du déplacement du masque, il est possible de déterminer la longueur d'onde de coupure à partir de laquelle l'inégalité (1) est respectée.

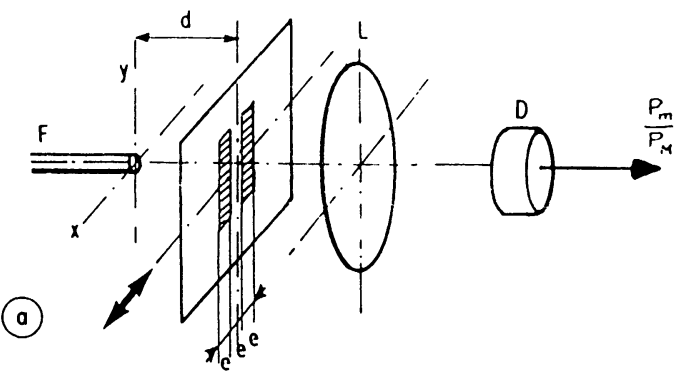

(b)

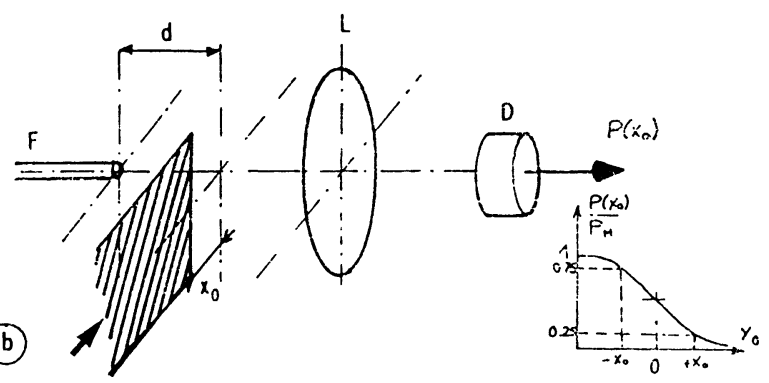

Fig. 7. - Dispositif de mesure de la dimension du mode, A : selon la méthode de Ronchi, B : selon la méthode de l'écran.

[Mode spot size measurement; A : Ronchi method, B : Knife edge method.]

Ces montages s'insèrent facilement sur les bancs d'atténuation spectrale et ne nécessitent aucune manipulation des extrémités de la fibre sous test.

Sur la figure 8 , nous présentons un résultat de 


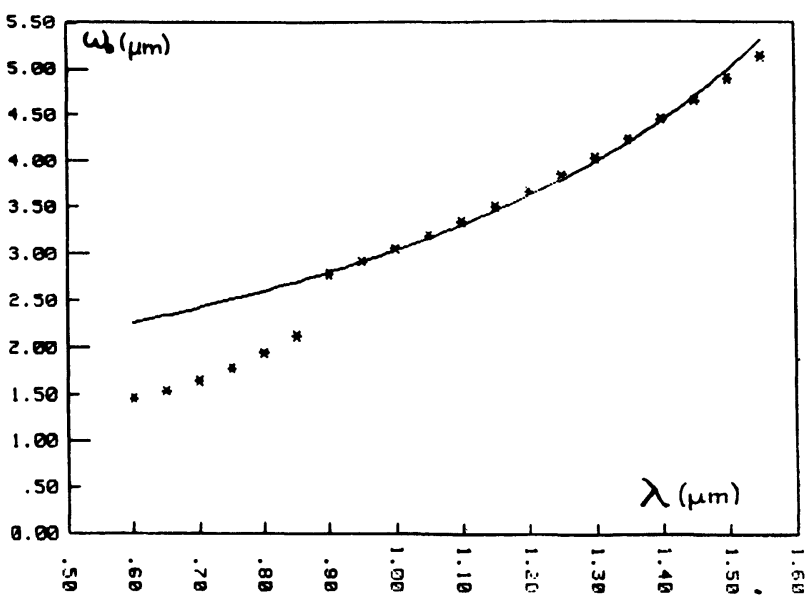

Fig. 8. - Evolution de la dimension du mode avec $\lambda$ et valeurs des paramètres de fibre équivalente $a_{\mathrm{e}}=2,52 \mu \mathrm{m}$, $\Delta n_{\mathrm{e}}=6,1 \times 10^{-3}, \lambda_{\mathrm{c}}=0,88 \mu \mathrm{m}$.

[Mode spot size versus wavelength curve and equivalent step index parameters.]

mesure donnant l'évolution de la dimension du mode en fonction de $\lambda$, utilisant un masque identique à celui présenté sur la figure $7 a$. A partir de ces données expérimentales, nous pouvons définir les paramètres équivalents à une fibre à échelon d'indice.

\section{Aptitude à la connexion et à l'épissurage.}

Si nous désignons par $\omega_{i}$ et $\omega_{j}$ les paramètres des modes correspondant aux deux fibres devant être raccordées, le facteur de transmission lié à la désadaptation modale s'écrit [15] :

$$
T=\left(\frac{2 \omega_{i} \omega_{j}}{\omega_{i}^{2}+\omega_{j}^{2}}\right)^{2}
$$

Ainsi, à la jonction de deux fibres $i$ et $j$ caractérisées par les paramètres : fibre $i \lambda_{\mathrm{c}}=1,1 \mu \mathrm{m}, \Delta n=5 \times 10^{-3}$ - fibre $j \lambda_{\mathrm{c}}=1,3 \mu \mathrm{m}, \Delta n=4 \times 10^{-3}$, la désadaptation modale se traduit par une perte de $0,1 \mathrm{~dB}$ à la longueur d'onde $\lambda=1,3 \mu \mathrm{m}$. Nous mesurons ainsi l'importance de la mesure de la dimension du mode ou de sa conservation durant le processus de fabrication.

A ces pertes viennent s'ajouter celles dues à un défaut de centrage du cour par rapport à la surface de référence naturelle que représente le diamètre extérieur de la fibre. Ainsi, pour deux fibres de mêmes caractéristiques optogéométriques et de diamètre de mode $7 \mu \mathrm{m}$, un défaut d'alignement de $0,5 \mu \mathrm{m}$ se traduit par une dégradation du facteur de transmission de $0,35 \mathrm{~dB}$.

Une méthode de mesure simple permettant d'avoir accès à ce défaut de centrage consiste à placer deux bouts de fibre issus de la même fibre mère dans un Vé. Le maintien mécanique des deux fibres dans le Vé étant effectué par aspiration. Une fibre constitue l'émetteur et l'autre la fibre réceptrice. La mesure du facteur de transmission en fonction de l'angle de rotation de la fibre réceptrice sur elle-même permet de remonter au paramètre d'excentrement du cour de la fibre.

Sur la figure 9, sont représentées les variations des pertes en fonction de l'angle de rotation de la fibre réceptrice présentant un défaut de centrage de $0,5 \mu \mathrm{m}$, la fibre ayant des caractéristiques optogéométriques $\operatorname{standard}\left(\lambda_{\mathrm{c}}=1,2 \mu \mathrm{m}, \Delta n=5 \times 10^{-3}, a=3,817 \mu \mathrm{m}\right)$.

Ces pertes sont calculées pour deux longueurs d'onde de source $\lambda=1,2 \mu \mathrm{m}$ et $\lambda=1,34 \mu \mathrm{m}$ mettant en évidence l'importance relative de ce défaut de centrage par rapport à la dimension du mode qui varie avec $\lambda$.

Il est à noter que la qualité de ce mode de caractérisation est étroitement liée à la préparation des extrémités de fibres mises en contact et à la stabilité de la source.

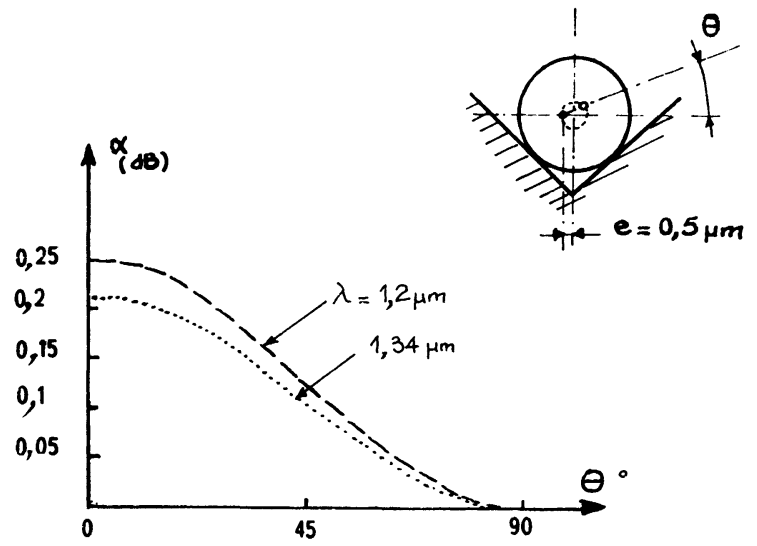

Fig. 9. - Variation du facteur de transmission au raccordement de fibres unimodales présentant un mauvais centrage du cœur.

[Splicing loss due to core cladding concentricity.]

\section{Sensibilité aux courbures.}

La sensibilité aux microcourbures des fibres optiques unimodales est étroitement liée à la structure de guidage et plus particulièrement à la dimension du mode.

Sur la figure 10, nous avons tracé les variations d'atténuation pour deux fibres lorsque celles-ci sont soumises à une perturbation sinusoïdale de leur axe [17].

Cette sensibilité peut par ailleurs être exploitée pour la détermination de la longueur d'onde de coupure $[18,19]$.

Un résultat de mesure (Fig. 11), correspondant à la variation d'intensité détectée en fonction de $\lambda$ lorsque la fibre est ou n'est pas soumise à une courbure, définit les différentes longueurs d'onde de coupure associées aux divers groupes de modes pouvant se propager. Cette expérimentation réalisée sur le même échantil- 


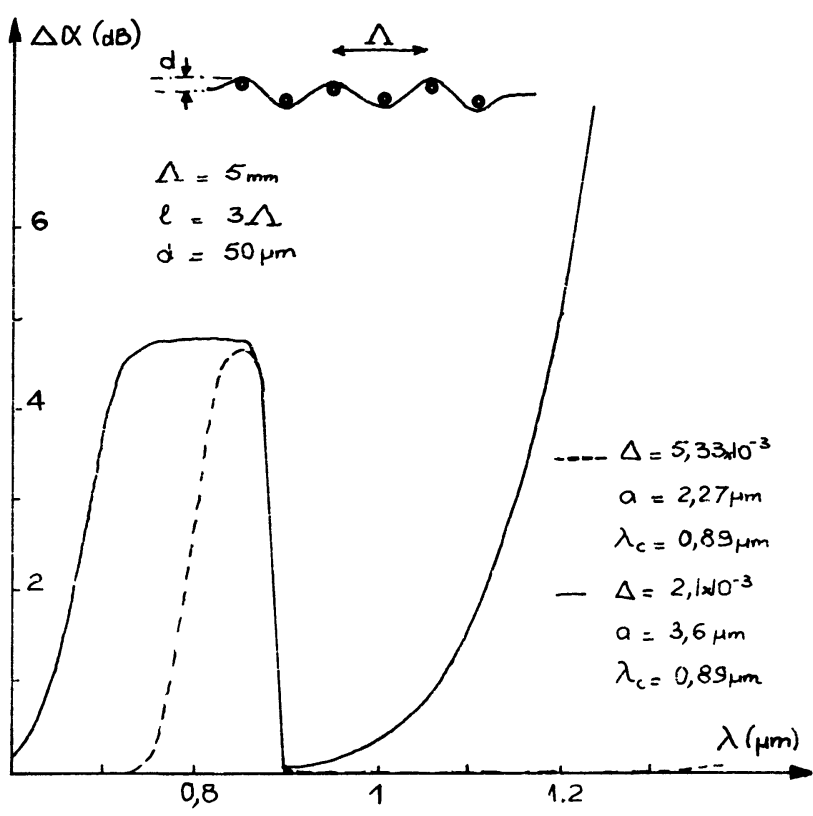

Fig. 10. - Influence de microcourbures sur la transmission en fonction des paramètres optogéométriques des fibres (calcul).

[Spectral bending losses versus the optogeometrical parameters of monomode fibres.]

lon que celui utilisé dans la détermination de la dimension du mode en fonction de $\lambda$, et présenté sur la figure 6, montre l'existence d'un bon accord sur la définition du régime monomode $(\lambda>0,88 \mu \mathrm{m})$.

\section{Dispersion.}

Les causes de dispersion dans les F.O.U. sont liées aux propriétés optogéométriques de la fibre (structure), au choix des matériaux et aux biréfringences de formes ou de contraintes qui viennent lever la dégénérescence associée au mode fondamental

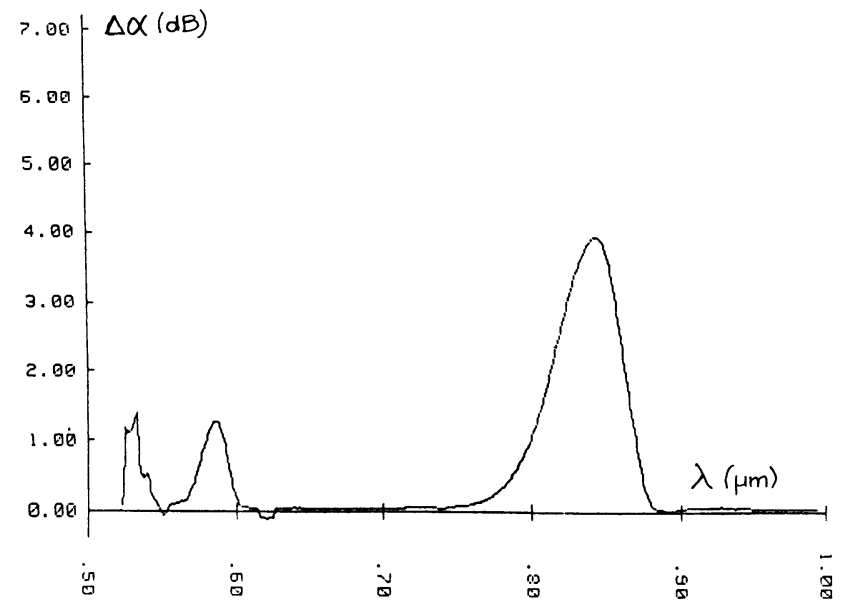

Fig. 11. - Détermination de la longueur d'onde de coupure sur une fibre optique par la méthode des courbures.

[Effective cut off wavelength obtained by measuring the higher order mode attenuations (bending method).]

pouvant se propager dans un guide diélectrique cylindrique.

Différentes méthodes furent mises au point, fondées sur la mesure de temps de propagation par l'emploi de sources accordables obtenues par génération d'effets non linéaires tels que l'effet Raman stimulé [20] ou par l'emploi de techniques d'interférométrie et d'étude de la cannelure du spectre [21, 22].

La figure 12 représente un montage de mesure du temps de groupe en fonction de la longueur d'onde. La source impulsionnelle et accordable de $\lambda=1,06 \mu \mathrm{m}$ à $1,8 \mu \mathrm{m}$ est obtenue en exploitant l'effet Raman stimulé généré dans une fibre monomode amorce. Un monochromateur sélectionné parmi le spectre Raman ainsi généré ... une composante spectrale impulsionnelle qui est couplée dans la fibre à tester.

La variation du temps de propagation de l'impulsion en fonction de $\lambda$ dans la fibre à caractériser est

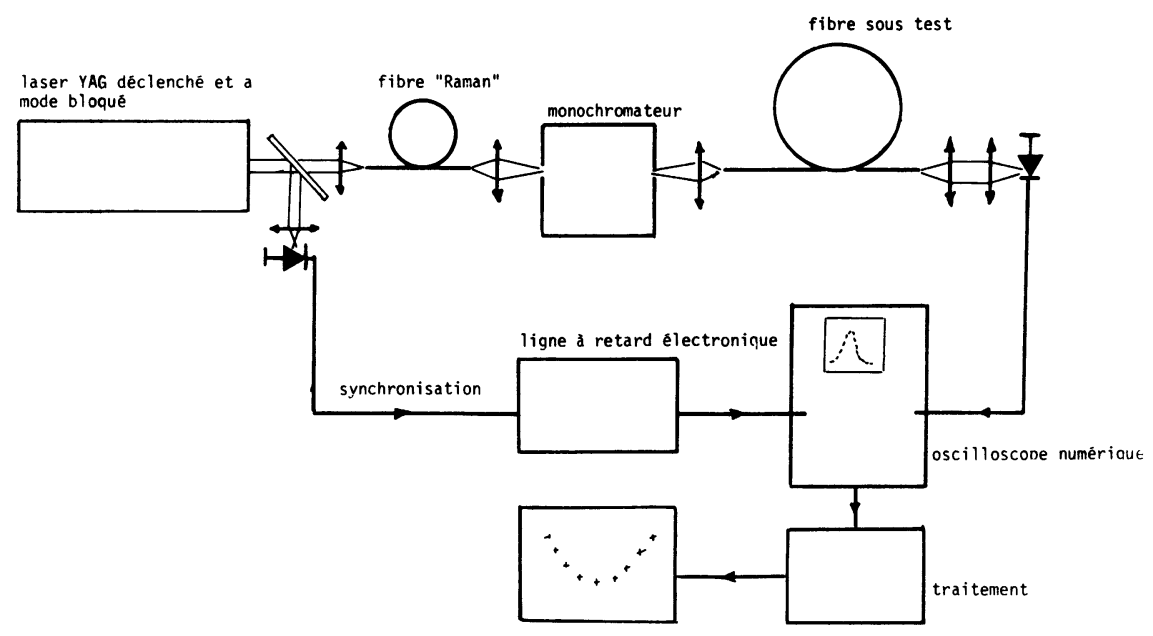

Fig. 12. - Schéma de montage de mesure de la dispersion chromatique.

[Experimental arrangement for relative delay time measurements (chromatic dispersion measurement).] 
fonction de la structure de guidage (dispersion intramodale) et du matériau utilisé pour réaliser la fibre (dispersion matériau).

La figure 13 donne le résultat de mesure ramené au $\mathrm{km}$ sur une fibre dont le profil d'indice est voisin de celui présenté sur la figure 4.

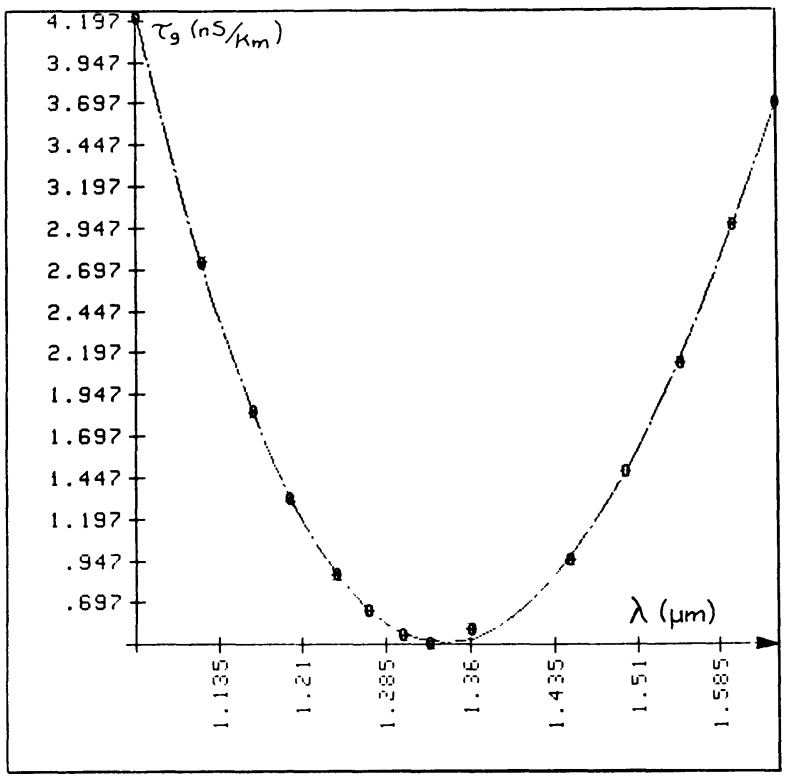

Fig. 13. - Résultat de mesure de la dispersion chromatique sur une fibre optique unimodale.

[Relative delay time versus wavelength measured on monomode fibre.]

En appliquant une méthode de régression polynomiale sur les points expérimentaux, le temps de propagation en fonction de $\lambda$ s'écrit :

$$
\tau_{\mathrm{g}}(\lambda)=\sum a_{i} \lambda^{i}
$$

En prenant la dérivée du temps de groupe, on détermine le coefficient de dispersion chromatique

$$
M(\mathrm{pS} / \mathrm{nm} . \mathrm{km})=\frac{\mathrm{d} \tau_{\mathrm{g}}}{\mathrm{d} \lambda}
$$

qui permet de définir la longueur d'onde optimale pour laquelle ce coefficient s'annule.

L'évolution du coefficient $M$ tiré des valeurs expérimentales (Fig. 13) donnant la variation du temps de groupe, correspond à la courbe $\mathrm{A}$ de la figure 14 .

Pour cette fibre, la longueur d'onde optimale $(M=0)$ est située à $1,34 \mu \mathrm{m}$. Ainsi, si le bilan fréquentiel l'exige, on a tout intérêt à centrer la source, devant être insérée dans la liaison, sur cette longueur d'onde optimale, ce qui permet de minimiser les effets dispersifs (élargissement impulsionnel).

La mesure $\tau_{g}(\lambda)$ ne permet pas de discriminer les effets liés au matériau de ceux correspondant à la dispersion du mode.

Dans l'approximation gaussienne des champs et

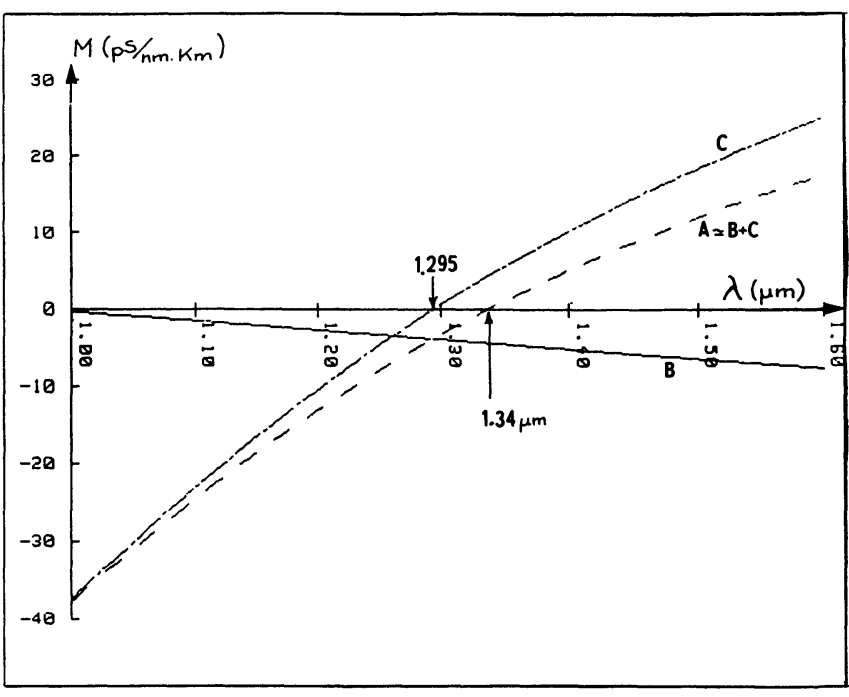

Fig. 14. - Variation du coefficient de dispersion. A : résultat de mesure par emploi d'une source Raman, B : dispersion intramodale calculée à partir de $\omega_{0}(\lambda), \mathrm{C}:$ dispersion matériau.

[Chromatic dispersion characteristics of monomode fibre ; A : total chromatic dispersion, B : modal dispersion computed from the spectral mode spot size dependence, $\mathrm{C}$ : material dispersion.]

pour une fibre à double indice, il est possible d'exprimer la dispersion modale à partir d'une mesure établissant la variation de la dimension du mode avec $\lambda[23,24]$ :

$$
\left.\frac{\mathrm{d} \tau_{\mathrm{g}}}{\mathrm{d} \lambda}\right|_{\bmod }=-\frac{\lambda}{\pi^{2} n_{2} c \omega_{0}^{2}}\left(\frac{\lambda}{\omega_{0}} \frac{\mathrm{d} \omega_{0}}{\mathrm{~d} \lambda}-\frac{1}{2}\right) .
$$

Sur la figure 14, la courbe B traduit la dispersion du mode en fonction de $\lambda$ calculée à partir d'une mesure de la dimension du mode $\omega_{0}(\lambda)$ selon la méthode décrite dans le $\S 4$.

Il est alors possible de remonter à la dispersion liée au matériau (courbe $\mathrm{C}$ ) et de définir sa longueur d'onde d'annulation. Pour ce type de fibre, elle se situe au voisinage de $1,29 \mu \mathrm{m}$; cette valeur est en bon accord avec une modélisation des propriétés dispersives de fibres à multi-dopants [25].

Par ailleurs, si l'on connaît les propriétés dispersives des matériaux utilisées, une simple mesure de la dimension du mode en fonction de $\lambda$ permet, par application de l'expression 10 , de contrôler la dispersion intramodale et de définir la longueur d'onde pour laquelle la dispersion chromatique est minimisée sans avoir à utiliser une source impulsionnelle accordable qui représente un lourd investissement.

\section{Conclusion.}

Dans la perspective d'emploi des fibres optiques unimodales dans les systèmes de transmission, nous avons décrit les différentes méthodes permettant de caractériser de tels composants. 
Il en ressort qu'une connaissance de la dimension du mode ou de sa variation avec $\lambda$ permet de définir :

- les pertes au raccordement,

- la sensibilité aux courbures ou microcourbures,

- la fibre à échelon d'indice équivalente,

- l'évolution de la dispersion intramodale,

- la micro-optique conjuguant au mieux la source à la fibre.

De plus, la grandeur de ce paramètre peut être évaluée sur un banc de mesure de l'atténuation spec- trale ce qui minimise la multiplication des bancs et la durée d'une caractérisation en production.

Durant notre exposé, les autres paramètres devant être mesurés seront classés selon un ordre hiérarchique dépendant des finalités de l'analyse des résultats (mise au point d'un banc de fabrication, définition de nouvelles structures de guides, étude d'une structure de câble ou d'un conditionnement particulier de la fibre, exploitation ou gestion d'un système monomode).

\section{Bibliographie}

[1] Jeunhomme, L., Fibres optiques monomodes pour les Télécommunications à grande distance et haut débit, Telemat 81, Marseille 25-27 mai 1981.

[2] Giallorenzi, T. G. et al., Optical Fiber sensor technology, IEEE Q.E. $18 \mathrm{n}^{\circ} 4(1982) 626$.

[3] Pocholle, J. P., TARDy, A., Mesures de grandeurs physiques fondées sur l'utilisation des fibres optiques, Conférence Européenne d'Optique 1980 «Horizons de l'Optique " Pont-à-Mousson 22-25/04/ 1980.

[4] ARditty, H. J., Capteurs à fibres optiques, Telemat 83, Marseille, 03/06/83.

[5] Papuchon, M., Puech, C., Les applications de l'optique intégrée, Telemat 81, Marseille, 25-27 mai 1981.

[6] Carenco, A., Les perspectives de l'optique intégrée, Telemat 81, Marseille, 25-27 mai 1981.

[7] Auge, J. et al., Mesure des profils d'indice de réfraction sur fibres et préformes, Rev. Tech. THOMSONCSF 13 n $^{\circ} 4$ (1981) 833.

[8] Stewart, W. J., A new technique for measuring the refractive index profils of graded optical fibres, 100C Tokyo, 18-20/07/1977.

[9] $\mathrm{CHu}$, P. L., Non destructive measurement of index profile of an optical fibre preform, Electron. Lett. 13 (1977) 736.

[10] Marcuse, D., Refractive index determination by the focusing method, Applied Opt. 18 (1979) 9.

[11] Sasaki, I., François, P. L., Payne, D. N., Accuracy and resolution of preform index profiling by the spatial filtering method, VIIth ECOC Copenhagen, September 1981.

[12] Millar, C. A., Direct method of determining equivalent step index profiles for monomode fibers, Electron. Lett. 17 (13) (1981) 458.

[13] Alard, F., Jeunhomme, L., Sansonetti, P., Fundamental mode spot size measurement in single mode fibers, Electron. Lett. 17 (25) (1981) 958.

[14] Pocholle, J. P., Auge, J., New simple method for measuring the mode spot size in monomode fibers, Electron. Letters 19 (6) (1983) 191.
[15] Marcuse, D., Loss analysis of single mode fibers splicing, Bell. Syst. Tech. J. 56 (5) (1977) 703.

[16] Jeunhomme, L., Single mode fiber design for long haul transmission, IEEE QE 18 (4) (1982) 727.

[17] Sansonetti, P., Pocholle, J. P., Gulllon, J., Comportement de F.O.U. sous l'effet de micro- et macrocourbures, 1er Colloque sur les transmissions monomodes, Paris, décembre 1980.

[18] Katsuyama, Y., Tokuda, M., Uchida, N., Nakahara, M., New method for measuring $V$ value of single mode optical fibre, Electron. Lett. 12 (25) (1976) 669.

[19] Gambling, W. A., Payne, D. N., Matsumura, H., NoRman, S. R., Measurements of normalized frequency in single mode optical fibres, Electron. Lett. 13 (5) (1977) 133.

[20] Cohen, Lg., Lin, C., Pulse delay measurements in the zero material dispersion wavelength region for optical fibres, Appl. Optics 16 (12) (1977) 3136.

[21] Shang, H. T., Chromatic dispersion measurements by white light interferometry on meter length single mode optical fibers, Electron. Lett. 17 (17) (1981) 603.

[22] Piasecki, J., Colombeau, B., Vampouille, M., FroEHLy, C., Subpicosecond coherent analysis of the impulse response of optical fibers, J. Optics 12 (11) (1981) 43.

[23] Sansonetti, P., Modal dispersion in single mode fibres : single approximation issued from mode spot size spectral behaviour, Electron. Lett. 18 (15) (1982) 647.

[24] STEWART, W. J., Understanding dispersion in monomode fibers, Opti. Fib. Comm. Phoenix 13-15/04/1982.

[25] Pocholle, J. P., Analyse de la dispersion chromatique dans les fibres optiques multimodes multidopants, Travaux non publiés. 